\title{
ANÁLISE DAS VARIÁVEIS MOTORAS E DA COMPOSIÇÃO CORPORAL EM LESADOS MEDULARES PRINCIPIANTES NA PRÁTICA DE ATLETISMO
}

Andréia Maria Micai Gatti

José Irineu Gorla

Adriana Nascimento de Souza

Mateus Betanho Campana

\section{Resumo}

Introdução: Este estudo teve por objetivo analisar as variáveis motoras e da composição corporal em indivíduos com Lesão Medular Traumática praticantes de atletismo. Materiais e Metodologia: Aplicou-se testes motores de agilidade, coordenação, força de preensão palmar e testes antropométricos. Foram avaliados cinco sujeitos que fazem parte do programa de reabilitação, modalidade atletismo, através da prática desportiva na cidade de Itú-SP. Resultados: Os resultados mostraram que na avaliação da composição corporal, quatro apresentavam níveis de gordura corporal acima dos referências normais. Discussão e Conclusão: Com relação a avaliação das variáveis motoras, o teste de batimento de placas para os Sujeitos do sexo masculino, a média foi de 12,37 seg., e para o sexo feminino foi de $17,45 \mathrm{seg}$. No teste de agilidade, a média para o sexo masculino foi de 19,98 seg., e o para o sexo feminino foi de 28,59 seg. O teste de Força de preensão palmar da mão direita e esquerda, a média dos Sujeitos do sexo masculino foi de $37,12 \mathrm{Kg}$ e $33,72 \mathrm{Kg}$ respectivamente, e para o sexo feminino foi de 9,6 Kg e 7,3 $\mathrm{Kg}$ respectivamente. Os resultados mostraram-se muito abaixo da média, segundo a literatura.

\section{Palavras-Chaves}

Traumatismos da Medula Espinal; Avaliação; Composição Corporal.

\section{ANALYSIS OF THE MOTOR VARIABLE AND THE BODY COMPOSITION IN INJURED MEDULARES BEGINNING IN THE PRACTICAL OF ATHLETICS}

\author{
Andréia Maria Micai Gatti \\ José Irineu Gorla \\ Adriana Nascimento de Souza \\ Mateus Betanho Campana
}

\begin{abstract}
The investigation, accompaniment and interpretation of various motor skills and the body composition of individuals with Spinal Cord Injury, can provide valuable information not only to describe the diagnosis of the situation, but also to identify and analyze determining factors for the establishment of measures of adequate intervention. The objective of this study is to analyze various motor skills and the body composition of individuals with Spinal Cord Injury who practice athletics. Five subjects who practice athletics were evaluated in the Municipal Clinic of Physiotherapy in the city of Itu/SP which comprised part of the rehabilitation program through the practice of sports. The evaluations done were tests of agility, coordination, force of palm pressure and anthropometric tests to evaluate body composition. The results showed that of the five subjects evaluated, in the evaluation of body composition, four presented levels of body fat above the normal standards. In relation to the motor skills, the test of beating the boards
\end{abstract}


for the male subjects, the average was 12.37 secs and the result for the female subjects was 17.45 secs. In the agility test, the average for the males was 19.98 secs and for the females was 28.59 secs. In the test of Palm Pressure of the right and left hand the average of the male subjects was $37.12 \mathrm{Kg}$ and $33.72 \mathrm{~kg}$ respectively. The results of the females were $9.6 \mathrm{Kg}$ and $7.3 \mathrm{Kg}$ respectively. The importance of evaluating body composition and the various motor skills of subjects with Spinal Cord Injury in the practice of sports was evident. The evaluation of body composition permitted us to prescribe physical activities which made for a better health condition together with a reduction in food intake. The evaluation of various motor skills, also permitted us to recommend physical training appropriate for the kind of tests which they practiced.

\section{Key-Words:}

Spinal Cord Injury, Evaluation, Body Composition. 


\section{Introdução}

A principal etiologia da lesão medular são as traumáticas, cerca de $80 \%$. Quanto ás causas traumáticas destaca-se o acidente automobilístico (principal causa no Brasil), o ferimento por arma de fogo (muito expressivo nos grandes centros urbanos com o aumento da violência - em São Paulo constitui a primeira causa) e ainda quedas, esportes e acidentes de trabalho entre outras. Em virtude da etiologia traumática altamente prevalente, a faixa etária mais acometida é a de jovens entre 18 e 40 anos, principalmente do sexo masculino (CRISTANTE, 2005).

A lesão medular constitui-se ainda hoje numa grave síndrome incapacitante, segundo Frontera (2003), a lesão da medula espinhal (LME) provoca inúmeras alterações neuromusculares, esqueléticas, hormonais, metabólicas e psicológicas no indivíduo lesionado.

Com relação à alteração músculoesquelética, Frontera (2003) afirma que a Lesão da Medula Espinhal leva a perda completa ou parcial do controle dos músculos inervados abaixo do nível de lesão, perda da força e resistência muscular e desmineralização óssea. A perda de força e resistência muscular inibe a resposta cardiorrespiratória ao exercício, uma vez que a fadiga local evita que os músculos mantenham as cargas de trabalho prescritas.

A paralisia musculoesquelética resulta em atrofia muscular. A atrofia dos músculos esqueléticos reduz a capacidade funcional para o exercício voluntário, uma vez que os movimentos primários abaixo do nível da lesão, tais como os músculos das pernas e/ou braços, não podem ser utilizados para exercícios.

A paralisia dos músculos abdominais e intercostais também diminui a capacidade fisiológica, pelo fato desses músculos auxiliarem com ventilação, a paralisia reduz a capacidade de alguém para ventilar energeticamente a performance atlética (FRONTERA, 2003).

As lesões no sistema simpático também dificultam a performance atlética. O sistema simpático influencia fortemente a performance, pois é responsável pela mobilização da energia armazenada, preparando o organismo para "lutar ou fugir".

Um sistema simpático deficiente diminui a resposta cardiovascular ao exercício e o efeito durante o esforço varia de acordo com o nível da lesão. Os sistemas simpáticos deficientes influenciam primariamente a performance atlética, afetando a regulação do músculo liso (por exemplo, prejudicando a 
capacidade de aumentar a pressão sanguínea) e diminuindo a capacidade da freqüência cardíaca aumentar durante uma atividade rigorosa (FRONTERA, 2003).

Associando as alterações neuromusculares, esqueléticas, hormonais, metabólicas e psicológicas decorrentes da Lesão medular traumática à inatividade crônica, observa-se o agravamento destas alterações.

A inatividade crônica é bem conhecida como tendo efeitos debilitantes sobre o estilo de vida, podendo aumentar a morbidade e a mortalidade mesmo em indivíduos aptos. O problema é mais agudo para os indivíduos com LME - o índice de mortalidade por doenças cardiovasculares é $228 \%$ mais alto do que na população em geral (KOCINA, 1997).

A inatividade física também pode levar á desmineralização óssea, à atrofia dos músculos esqueléticos e cardíaco, à diminuição da massa corporal magra, à redução do conteúdo de água corporal e do volume sanguíneo e aumento da gordura corporal. (FIGONI, 1993).

A deficiência leva a uma diminuição na capacidade de trabalho em graus variados. Recentes estudos mostram que as causas de morte em lesados medulares estão se aproximando daquelas da população em geral, com aumento na prevalência de doenças cardiovasculares e outras associadas ao sedentarismo. Os efeitos do sedentarismo, tais como diminuição da resistência aeróbica, da força e flexibilidade muscular, somados à deficiência, levam a uma perda de capacidade funcional e independência que poderia ser parcialmente evitada. A prática desportiva neste grupo tem impacto positivo mais evidente e critico que em indivíduos normais (GHORAYEB, 1999).

A importância do exercício regular e da participação em esportes para reabilitação de indivíduos com lesão da medula espinhal tem sido reconhecida desde a Segunda Guerra Mundial. A despeito dessa conscientização, há pouca compreensão de como o exercício pode influenciar a qualidade de vida dos indivíduos com LME (NOREAU; SHEPHARD, 1992).

O esporte terapêutico é definido pela Federação Alemã de Desporto para a Saúde e Desporto Terapêutico como sendo um meio de terapia pelo movimento, que se utiliza de recursos adequados do desporto, 
visando compensar ou regenerar distúrbios funcionais de ordem física, psíquica e social, prevenir contra distúrbios secundários e promover um comportamento orientado para a saúde (SCHÜLE, 1988).

Paeslack (1978) é de opinião que todo paraplégico necessita de terapia esportiva, independente da idade, do sexo, da altura da lesão, das suas causas e de eventual presença de outros ferimentos.

A terapia esportiva não deve ser encarada como um esporte de lazer, praticado uma vez por semana por um determinado tempo, e sim uma exigência terapêutica, que deve ser satisfeita diariamente.

Sarrias (1976) é de opinião que o esporte não é apenas uma atividade recreativa, mas sim um ótimo suplemento à fisioterapia. Tem, além disso, um grande valor na reabilitação psicológica e social do tetraplégico.

Segundo Souza (1994), os objetivos do desporto para paraplégicos e tetraplégicos são: favorecer a transição entre a deficiência e a aptidão; promover a educação para a saúde; facilitar o reingresso da pessoa na sua vida familiar, social, educacional, profissional e no lazer ativo; tornar os indivíduos mais independentes, reduzindo a dependência física e psíquica; aprimorar a técnica de manejo de cadeira de rodas; promover, quando for o caso, a iniciação e a prática desportiva entre outras.

Segundo Greve, Casalis e Barros Filho (2001), diferentemente do exercício, que pressupõe um esforço localizado ou generalizado voltado para o preparo físico e, mais ainda, da atividade física que pressupõe a organização de séries de exercícios voltados para o combate ao sedentarismo e manutenção da qualidade de vida, o movimento voltado para o esporte ou atividade esportiva pressupõe que se superarem os prérequisitos coordenação, condição funcional, condicionamento físico com preparo cardiovascular e musculoarticular.

Para pessoas com lesão medular que buscam uma atividade física, os objetivos principais devem ser a manutenção da boa saúde e a melhora da aptidão física. O professor deve inicialmente avaliar seu aluno a fim de detectar possíveis problemas, como a falta de flexibilidade; a incapacidade de sustentar atividade aeróbica; a falta de força e resistência para erguer o corpo, transferi-lo de forma independente, ou para erguer o corpo para prevenir úlceras de decúbito e impulsionar a cadeira de rodas; sendo a porcentagem 
de gordura excessiva incompatível com uma boa saúde e seu controle uma necessidade suprida pela avaliação física periódica (GORGATTI; COSTA, 2005).

Diversos são os critérios para que um atleta dito normal possa habilitar-se para competir, tais como: gênero, idade, se é atleta profissional, dentre outros.

Desta forma, quando se fala em desporto para pessoas com deficiência, o princípio de "justiça" está em separar os desportistas segundo seus diferentes e distintos graus de deficiência, para que se possa estabelecer um equilíbrio ou nivelamento das ações motoras, procurando assegurar na medida do possível, condições de igualdade próximas das ideais. Sendo a classificação funcional uma ferramenta eficaz para este nivelamento.

A Classificação Funcional consiste em uma categorização, em que o atleta recebe em função de seu volume de ação, ou seja, de sua capacidade de realizar movimentos, colocando em evidência a potencialidade motora dos resíduos musculares da seqüela de algum tipo de deficiência, bem, como, os músculos que não são lesados.

Segundo Gorla (1997), os objetivos das medidas e avaliações são: determinar o progresso do indivíduo; classificar e selecionar indivíduos; diagnosticar; motivar; estabelecer e reciclar o programa de treinamento; acompanhar o processo de crescimento e desenvolvimento dos alunos; detectar deficiências, permitindo uma orientação no sentido de superá-los; além de servirem como diretrizes para pesquisas.

Adaptações diversas para avaliação do deficiente são necessárias, porém a maior dificuldade ainda são os índices e tabelas. Neste momento em que se inicia a implantação da avaliação cineantropométrica para indivíduos em cadeiras de rodas o paradigma clássico que compara o indivíduo com tabelas referenciais não deve ser observado (MELLO, 2003).

Alguns autores citam estudos que examinam a questão da análise da composição corporal em portadores de lesões medulares. Por não apresentarem mobilidade em grande parte do corpo, esses indivíduos têm tendência ao acúmulo de gordura e à menor proporção de massa magra (muscular e óssea). Um problema para a realização desse tipo de estudo é o número de indivíduos disponíveis nas amostras, principalmente quando se trata de atletas (KISS, 2003). É comum o professor de educação física empregar estratégicas de 
análise da eficiência do ensino (avaliação) quando inicia um programa de intervenção, durante e depois para confirmar se os objetivos foram alcançados (CASTRO, 2006).

Como forma de suprir esta carência de estudos e propiciar referencial para novas intervenções este trabalho teve por objetivo analisar as variáveis motoras e da composição corporal em indivíduos com Lesão Medular Traumática praticantes de atletismo.

Para atingir este objetivo foram aplicados testes motores para avaliar agilidade, coordenação, força de preensão palmar e testes antropométricos para avaliar a composição corporal.

A investigação, o acompanhamento e interpretação de variáveis motoras e da composição corporal em indivíduos com Lesão Medular Traumática, podem fornecer informações valiosas não somente para descrever um diagnóstico de situação, mas também para proporcionar a identificação e análise de fatores determinantes para o estabelecimento de medidas de intervenção adequadas.

\section{Metodologia}

O presente estudo de caso teve como população cinco Sujeitos com Lesão Medular Traumática que iniciaram a prática de atletismo há seis meses. Todos fazem parte do programa de reabilitação através da prática desportiva da Clínica Municipal de Fisioterapia de Itu/SP. Conforme o quadro 1, dos cinco Sujeitos com Lesão Medular Traumática, quatro são do gênero masculino e um do gênero feminino. Lembrando que a incidência de Lesão Medular Traumática é predominantemente do sexo masculino.

A participação feminina ainda que discreta na prática desportiva vem crescendo e este estudo registra esta participação feminina para contribuição e informação para futuros estudos.

A caracterização do grupo amostral é apresentada no quadro 1 : 


\section{QUADRO 1- Caracterização do Grupo Amostral}

\begin{tabular}{|c|c|c|c|c|c|c|c|}
\hline Sujeito & Gênero & Idade & $\begin{array}{c}\text { Nível } \\
\text { da } \\
\text { Lesão }\end{array}$ & $\begin{array}{l}\text { Tipo de } \\
\text { Secção }\end{array}$ & $\begin{array}{c}\text { Tempo } \\
\text { de } \\
\text { Lesão }\end{array}$ & $\begin{array}{c}\text { Modalidade } \\
\text { Atletismo }\end{array}$ & $\begin{array}{c}\text { Classificação } \\
\text { Funcional }\end{array}$ \\
\hline $\mathrm{I}$ & M & $\begin{array}{c}31 \\
\text { anos }\end{array}$ & $\mathrm{T} 7$ & incompleta & 10anos & Corrida $400 \mathrm{mts}$. & Т 53 \\
\hline II & M & $\begin{array}{c}28 \\
\text { anos }\end{array}$ & $\mathrm{T} 7$ & completa & 10anos & Arremesso de peso & F54 \\
\hline III & $\mathrm{F}$ & $\begin{array}{c}54 \\
\text { anos }\end{array}$ & C5 & incompleta & 7 anos & Lançamento de disco & F53 \\
\hline IV & M & $\begin{array}{c}33 \\
\text { anos }\end{array}$ & $\mathrm{T} 5$ & completa & 6 anos & $\begin{array}{l}\text { Lançamento de disco } \\
\text { e } \\
\text { Corrida de 100mts. }\end{array}$ & $\begin{array}{l}\text { F53 } \\
\text { T53 }\end{array}$ \\
\hline$V$ & M & $\begin{array}{c}48 \\
\text { anos }\end{array}$ & $\mathrm{C} 5$ & incompleta & 15 anos & $\begin{array}{c}\text { Lançamento de disco } \\
\text { e } \\
\text { Corrida } 100 \mathrm{mts} .\end{array}$ & $\begin{array}{l}\text { F53 } \\
\text { T53 }\end{array}$ \\
\hline
\end{tabular}

F(field): Campo 53: Apresentam total força de ombro e punho. Têm força quase total de flexores e extensores de dedos.Possuem musculatura intrínseca das mãos funcionais, porém não normais.

F (field): Campo 54: Tem função normal de membros superiores, possuem músculos abdominais e extensores de coluna funcionais.

T (track): Pista 53: Apresentam função normal de membros superiores. Músculos abdominais superiores bons e músculos abdominais inferiores sem uso.

Os instrumentos utilizados para avaliar as medidas antropométricas foram o Compasso de Dobras Cutâneas da Marca CESCORF, a Fita Antropométrica da Marca Cardiomed, a balança digital da marca Filizola.

Quanto aos procedimentos, para as medidas de dobras cutâneas, foi aferida a espessura da dobra cutânea na região subescapular no hemi-corpo direito, sendo que os indivíduos foram orientados a permanecer em postura ereta com os membros superiores paralelos ao tronco.

A circunferência abdominal foi aferida com os indivíduos sentados em suas próprias cadeiras, com a fita colocada à altura da cicatriz umbilical. A circunferência da panturrilha foi aferida à altura medial da tíbia. O diâmetro ósseo do tórax foi aferido à altura do mamilo. 
Para análise dos dados coletados foram utilizadas duas equações: a primeira, proposta por Bulbulian et al. (1987), desenvolvida para estimar a densidade corporal, e a segunda proposta por Siri (1961) para estimar o percentual de gordura.

Sendo: Densidade corporal $=1,09092+0,00296$ (diâmetro torácico, em cm) - 0,00072 (dobra cutânea subescapular, em mm) - 0,00182 (circunferência abdominal, em cm) + 0,00124 (circunferência da panturrilha medial, em $\mathrm{cm}$ ). (Erro padrão de estimativa $=0,0064)$, e para a estimativa do percentual de gordura será utilizada a equação proposta por SIRI (1961), sendo: \%Gord = (4.95/Dens - 4.50)100.

Para análise dos dados será utilizada a estatística descritiva (Média e desvio padrão), através de um programa computadorizado versão Biostat 3.0.

Com relação as variáveis motoras os instrumentos utilizados foram um dinamômetro da marca Filizola, modelo Crown, para o teste de força, aplicado com o avaliado sentado, com o membro superior estendido na lateral do corpo, segurando o aparelho pela empunhadeira e realizando uma flexão máxima dos dedos. Foram coletadas três medidas para cada uma das mãos, sendo utilizado a média neste estudo.

O teste de agilidade (teste de zigue-zague modificado) foi aplicado conforme metodologia sugerida por Gorgatti e Costa (2005).

Para a realização do teste de Batimento em placas foram utilizados um cronômetro com definição centesimal, mesa adaptada à altura do avaliado, dois círculos autocolantes com $20 \mathrm{~cm}$ de diâmetro cada um fixados sobre a mesa e distantes $80 \mathrm{~cm}$ dos seus centros e um retângulo também autocolante com dimensões $10 \mathrm{~cm}$ x $20 \mathrm{~cm}$ colocado eqüidistante entre ambos os círculos. O avaliado permaneceu sentado na sua cadeira de rodas de frente para a mesa, com a mão não dominante apoiada no retângulo central sobre a mesa. A mão dominante iniciou o teste apoiada no círculo fixado do lado oposto que resultará em cruzamento dos braços.

Ao sinal sonoro emitido pelo avaliador, o avaliado moveu a mão dominante, tocando no círculo oposto o mais rápido possível, passando por cima da mão não dominante colocada no meio, que permaneceu apoiada no retângulo de origem, completando dessa forma um ciclo. O teste consistiu em realizar 25 
ciclos completos (50 toques alternadamente nos círculos) no menor tempo possível. O cronômetro foi acionado ao sinal sonoro do avaliador e travado assim que o avaliado completou os 25 ciclos.

\section{Análise e Discussão de Dados}

Os dados são apresentados em dois grupos, divididos por gênero: Grupo I - Sexo Masculino (4) e Grupo II - Sexo Feminino.

A Tabela 1 apresenta os resultados das Variáveis Antropométricas, com média e desvio padrão do Grupo I (masculino), enquanto a tabela 2 faz referência ao Grupo II (feminino).

Tabela 1- Variáveis Antropométricas - Masculino

\begin{tabular}{|c|c|c|c|c|}
\hline Sujeito & Lesão & Idade & $\begin{array}{l}\text { MC* }^{*} \\
(\mathrm{~kg})\end{array}$ & $\begin{array}{c}\text { Estatura } \\
\text { (cm) }\end{array}$ \\
\hline I & $\mathrm{T} 7$ & 31 & 57,8 & 179 \\
\hline II & T7 & 28 & 65,8 & 178 \\
\hline III & T5 & 33 & 74,2 & 180 \\
\hline IV & $\mathrm{C} 5$ & 48 & 66,8 & 173 \\
\hline Média & & 35 & 66,15 & 177,5 \\
\hline SD & & $\pm 7,71$ & $\pm 5,81$ & $\pm 0,02$ \\
\hline
\end{tabular}

Tabela 2- Variáveis Antropométricas - Feminino

\begin{tabular}{ccccc}
\hline Sujeito & Lesão & $\begin{array}{c}\text { Idade } \\
\text { (anos) }\end{array}$ & $\begin{array}{c}\text { MC } \\
\text { (kg) }\end{array}$ & $\begin{array}{c}\text { Estatura } \\
\text { (cm) }\end{array}$ \\
\hline$I$ & C5 & 54 & 82,4 & 168
\end{tabular}

A Tabela 1- apresenta aparentemente uma correlação boa entre massa corporal e estatura. O Sujeito I apresenta claramente um peso abaixo do normal.

A Tabela 2- apresenta claramente um sobrepeso do Sujeito I considerando a correlação estatura e massa corporal.

As Tabelas 3 e 4 apresentam os resultados das medidas de composição corporal de Diâmetro Torácico (DT), Circunferência Abdominal (CA), Circunferência de Perna (CP), Dobra Cutânea Sub-escapular 
(SE), utilizados para o cálculo de Densidade Corporal (DC), Percentual de Gordura (\%G) e Erro Padrão (EP).

Tabela 3 - Composição Corporal - Masculino

\begin{tabular}{ccccccc}
\hline Sujeito & $\begin{array}{c}\text { DT } \\
(\mathbf{c m})\end{array}$ & $\begin{array}{c}\text { CA } \\
(\mathbf{c m})\end{array}$ & $\begin{array}{c}\text { CP } \\
(\mathbf{c m})\end{array}$ & $\begin{array}{c}\text { SE } \\
(\mathbf{m m})\end{array}$ & D.C & \%G \\
\hline I & 28,2 & 73,7 & 28,5 & 10,2 & 1,06 & 13,37 \\
II & 30,5 & 86 & 25,6 & 10,6 & 1,04 & 21,97 \\
III & 31,5 & 106,5 & 31,4 & 31,5 & 1,00 & 41,76 \\
IV & 30,7 & 97 & 32 & 20,4 & 1,03 & 30,46 \\
\hline Média & 30,22 & 90,8 & 29,37 & 18,1 & 1,03 & 26,89 \\
EP & $\pm 0,54$ & $\pm 5,4$ & $\pm 1,14$ & $\pm 3,89$ & $\pm 0,01$ & $\pm 4,69$ \\
\hline
\end{tabular}

DT - Diâmetro Torácico; CA - Circunferência Abdominal; CP - Circunferência Panturrilha;

SE - Dobra Cutânea Subescapular; DC - Densidade Corporal; \%G - Percentual de Gordura.

Tabela 4 - Composição Corporal - Feminino

\begin{tabular}{ccccccc}
\hline Sujeito & DT(cm) & CA(cm) & CP(cm) & $\begin{array}{c}\text { SE } \\
(\mathbf{m m})\end{array}$ & D.C & \%G \\
\hline $\mathrm{V}$ & 29,2 & 100 & 37 & 37 & 1,01 & 37,88
\end{tabular}

DT - Diâmetro Torácico; CA - Circunferência Abdominal; CP - Circunferência Panturrilha; SE - Dobra Cutânea Subescapular; DC - Densidade Corporal; \%G - Percentual de Gordura.

Observando a Tabela 3 é possível afirmar que, dos cinco Sujeitos, quatro apresentaram níveis de gordura corporal acima dos referenciais normais. As referências de percentual de gordura para lesados medulares, vem sendo alvo de estudos, porém ainda não existem parâmetros para esta população. Por isso foram utilizados parâmetros de referência estabelecidos para populações sem deficiência.

Segundo valores estimativos para gordura corporal, o nível recomendado para homens é de $15 \%$ e para mulheres $25 \%$, enquanto a quantidade de gordura de $25 \%$ ou mais para homens e $32 \%$ ou mais para mulheres são considerados como obesidade (HEYWARD; STOLARCZYK, 1996).

Estudos sobre composição corporal em Sujeitos com deficiência são recentes e muitas vezes utilizam adaptações dos métodos convencionais aplicados aos indivíduos normais. Contudo, quando se trata de pessoas com deficiências físicas, em alguns casos, tais métodos podem ser seriamente comprometidos (KISS, 2003). 
Técnicas como bioimpedância, pesagem hidrostática e soma de dobras cutâneas são utilizadas como parâmetros para a determinação da composição corporal desses Sujeitos. Contudo, a simples adaptação desses métodos não elimina o erro natural em que se incorre quando não se leva em consideração a elevada percentagem de gordura que esses Sujeitos certamente terão nos membros inferiores e na região do tronco, abaixo do nível da lesão (KISS, 2003).

Figoni, Lockette e Surburg (1995) citam estudos que examinam a questão da análise da composição corporal em pessoas com lesões medulares. Por não apresentarem mobilidade em grande parte do corpo, esses Sujeitos têm tendência ao acúmulo de gordura e à menor proporção de massa magra (muscular e óssea).

Alguns autores alertam que a coleta de dobras para pessoas com deficiências físicas pode ser um método interessante por fornecer uma indicação de onde a gordura corporal pode ser mais concentrada nesta população (WELLS; HOOKER, 1990).

A tendência atual dos pesquisadores é buscar validar equações que possam predizer com maior fidedignidade a composição corporal de Sujeitos com deficiência física. A equação proposta por Bulbulian et al. (1987) apresenta um $r=0,95$ e um erro padrão de estimativa $=0,0064$.

Torna-se importante observar que esta equação foi validada apenas para os atletas paraplégicos do sexo masculino dos Estados Unidos e que sua extrapolação para outras populações dependeria de mais estudos.

\section{Tabela 5- Variáveis Motoras - Masculino}

\begin{tabular}{ccccc}
\hline SUJEITO & BP(seg.) & AGILIDADE(seg.) & FPMD(kg) & FPME(kg) \\
\hline I & 13,36 & 18,43 & 45,3 & 38,3 \\
II & 11,37 & 18,99 & 54,3 & 45,0 \\
III & 12,27 & 19,23 & 42,6 & 31,0 \\
IV & 12,49 & 23,29 & 6,3 & 20,6 \\
\hline Média & 12,37 & 19,98 & 37,12 & 33,72 \\
\hline EP & $\pm 0,40$ & $\pm 1,11$ & $\pm 10,57$ & $\pm 5,22$ \\
\hline
\end{tabular}

BP - Batimento de Placas; FPMD - Força de Preensão Palmar Mão Direita; FPME - Força de Preensão Palmar Mão esquerda; EP - Erro Padrão 
Tabela 6 - Variáveis Motoras - Feminino

\begin{tabular}{ccccc}
\hline Sujeito & $\begin{array}{c}\text { BP } \\
\text { (seg.) }\end{array}$ & $\begin{array}{c}\text { AGILIDADE } \\
\text { (seg.) }\end{array}$ & $\begin{array}{c}\text { FPMD } \\
\text { (seg.) }\end{array}$ & $\begin{array}{c}\text { FPME } \\
\text { (seg.) }\end{array}$ \\
\hline $\mathrm{V}$ & 17,45 & 28,59 & 9,6 & 7,3
\end{tabular}

BP - Batimento de Placas; FPMD - Força de Preensão Palmar Mão Direita;

FPME - Força de Preensão Palmar Mão Esquerda.

$\mathrm{O}$ teste de Batimentos de Placas tem por objetivo associar o componente motor à velocidade de membros superiores, sendo possível observar que o menor tempo (melhor desempenho) foi conquistado pelo Sujeito II.

Apesar de não existirem parâmetros na literatura, em tese os Sujeitos I, II, III, por serem paraplégicos, deveriam apresentar um menor tempo com relação ao Sujeito IV (tetraplégico incompleto), o que não ocorreu com o Sujeito I, além do desvio padrão ser pequeno entre os Sujeitos, podendo o fator tempo de lesão ser a variável para este resultado.

O Sujeito V fez o maior tempo, comparado com os Sujeitos do sexo masculino, sugere-se que tenha havido interferência quanto ao sexo, idade e nível da lesão.

Com relação ao teste de agilidade modificada, segundo Sale (1991), existem quatro razões para se testar a agilidade: determinar sua relevância no desempenho, desenvolver o perfil do atleta, monitorar a reabilitação de lesões.

Nos estudos realizados para autenticidade científica de um teste de agilidade para indivíduos em cadeiras de rodas, Gorgatti e Böhme (2003), mostram os seguintes resultados para indivíduos do sexo masculino, lesados medulares completos, variando entre T10 - L4, jogadores de basquete e sedentários. Para atletas, o tempo médio foi de 14,6 segundos e para os sedentários o tempo médio foi de 24,9 segundos.

No entanto os estudos realizados para análise das variáveis motoras em atletas de basquetebol em cadeiras de rodas, mostram os seguintes resultados, para indivíduos do sexo masculino, com seqüela de poliomielite, um tempo médio de 13,45 segundos (GORLA; ARAUJO; CARMINATO, 2005). 
O tempo médio dos indivíduos do sexo masculino, lesados medulares com nível de lesão entre C5-T7, iniciantes na prática de atletismo foi de 19,9 segundos, conforme Tabela 5.

O Sujeito V do sexo feminino, lesão medular, nível C5 incompleta, obteve um tempo médio de 28,59 segundos. Sugere-se levar em conta o sexo, fator idade e nível da lesão e a modalidade praticada e o tipo de prova (arremesso de peso e lançamento de disco).

Com relação à Força de preensão palmar direita e esquerda, foi possível observar que os Sujeitos obtiveram um melhor desempenho com a mão direita, exceto o Sujeito IV que apresentou um resultado muito abaixo, se comparado ao grupo de pessoas ditas normais. Provavelmente essa interferência deve-se ao nível da lesão e com o tipo de lesão (afetando mais o lado direito).

O Sujeito V do sexo feminino apresentou um resultado muito abaixo do esperado, este resultado quando analisado em relação aos padrões normais, pode ser considerado muito fraco. Sugere-se que fatores como sexo, tipo de lesão, nível de lesão e fator idade, pois para Corbin (1991), para pessoa acima de 50 anos, pode reduzir os escores em 5\% a 10\% em função do tecido muscular devido ao envelhecimento.

Estes valores mostram que há necessidade de um melhor desenvolvimento da força manual para essa população, visto a importância e utilidade desta para o manejo da cadeira de rodas.

\section{Referências}

APPELL, H. J ; MAURITZ, K. H. Sport in der Rehabilitation, Sant Augustin, p. 25-42, 1988. ????? BUlBulian, R. et al. Body composition in paraplegic male athletes. Med Sci Sports Exerc. v. 19, n. 3, p. 195-201, 1987.

CASTRO, E. M. Atividade motora adaptada. São Paulo: Tecmedd, 2006. 
CORBIN, C. B. A Multidimensional hierarchical model of physical fitness: A basis for integration and collaboration. Quest, v. 43, p. 296-306, 1991.

CRISTANTE, A. R. L. Aspectos clínicos. In: Moura, E. W.; SILVA, P. A. C. Fisioterapia: aspectos clínicos e práticos da reabilitação. São Paulo: Artes Médicas, 2005. cap. 8.1, p. 501-502.

FIGONI, S. F. Exercise responses and quadriplegia. Med Sci Sports Exerc. v. 25 p. 433-41. 1993.

.; LOCKETTE, K. F; SURBURG, P. R. Exercise prescription: adapting principles of conditioning. In: MILLER, P. D. (Ed.). Fitness programming disability. Champaing: Human Kinetics, 1995, p. 67-77.

FRONTERA, W. R.; DAWSON, D. M.; SLOVIK, D. M. Exercício físico e reabilitação. São Paulo: Artmed, 2003.

GHORAYBEB, N. O exercício: preparação fisiológica, avaliação médica aspectos especiais e preventivos. São Paulo. Atheneu, p. 321-366. 1999.

GORGATTI, M. G.; BÖHME, M. T. S. Autenticidade científica de um teste de agilidade para indivíduos em cadeira de rodas. Revista Paulista de Educação Física. São Paulo, n. 17, n. 1, p. 41-50, jan/jun. 2003.

.; COSTA, R. F. Educação Física adaptada. São Paulo: Manole, 2005.

GORLA, J. I. Educação Física especial: testes. Rolândia, Paraná, 1997.

.; ARAUJO, P. F.; CARMINATO, R. A. Análise das variáveis motoras em atletas de basquetebol em cadeiras de rodas. Revista Digital, <http://www.efdeportes.com/efd83/cadeiras.htm>. Buenos

Aires, n. 83, abr. 2005. Acesso em: 05 fev. 2007.

GREVE, J. M. A.; CASALIS, M. E. P.; BARROS FILHO, T. E. P. Diagnóstico e tratamento da lesão da medula espinal. São Paulo: Roca, 2001.

HENSCHEL, A.. Techniques for measuring body composition. Washington: National Academy of Science, 1961. p. 223-224

HEYWARD, V. H; STOLARCZYK, L. M. Applied body composition assessment. Champaign: Human Kinetics, 1996.

KISS, M. A. P. Esporte e exercício: avaliação e prescrição. São Paulo: Roca, 2003.

KOCINA, P. Body composition of spinal cord injured adults. Sports Med., n. 23, p. 48-60, 1997.

MELLO, M. T. Paraolimpíadas Sidney 2000: avaliação do treinamento dos atletas brasileiros. São Paulo: Atheneu, 2002.

NOREAU, L.; SHEPHARD, R. J. Return to work after SCI: the potential contribution of physical fitness.

Paraplegia n. 30, p. 563-72, 1992. 
PAESLACK, V. Esporte para os paraplégicos. In: HUELLEMANN, K. D. Medicina esportiva: clínica e prática, São Paulo: EPU, 1978.

SALE, D. G. Testing strength and power. In: MacDOUGALL, V. D.; WENGER, H. A.; GREEN, H. J. Physiological testing of high performance athlete. Champaing: Human Kinetics, 1991. p. 21-106.

SARRIAS, M. D. Rehabilitación del tetrapléjico espinal. In: GONZALEZ, M. A. S. Tratado de rehabilitación médica. Barcelona: Científico-Médica, 1976

SCHÜLE, K. Die Stellung der Sporttherapie und des Sporttherapeuten in der Rehabilitationskette. In:

SOUZA, P. A. O esporte na paraplegia e tetraplegia. Rio de Janeiro: Guanabara Koogan, 1994.

SIRI, W. E. Body composition from fluid spaces and density: analysis of methods. In: BROZEK, J.;

WELLS, C. L.; HOOKER, S. P. The spinal injuried athete. Adapted Phisical Activity Quarterly, v. 7, p. $265-285,1990$.

GORLA, J. I; ARAUJO, P. F; CARMINATO, R. A.; Análise das Variáveis Motoras em Atletas de Basquetebol em Cadeiras de Rodas. Revista Digital. Buenos Aires: n. 83, abril, 2005. <http://www.efdeportes.com/efd83/cadeiras.htm>. Acesso em: 05 fev. 2007. 


\begin{abstract}
Andréia Maria Micai Gatti
Faculdade de EducaçãoFísica/UNICAMP

Professora Especialista, Membro do Grupo de Estudos em Atividade Motora Adaptada GEPAMA

Fisioterapeuta- Clinica Municipal de Itu
\end{abstract}

\title{
José Irineu Gorla
}

Faculdade de Educação Física/UNICAMP

e-mail gorla@fef.unicamp.br

Fone 19- 35216616

Doutor do Depto de Estudos da Atividade Física Adaptada

Coordenador da Área de Educação Física da Federação das Entidades Assistenciais de Campinas

Fundação Odila e Lafayette Álvaro

\section{Adriana Nascimento de Souza}

Faculdade de EducaçãoFísica/UNICAMP

Professora Especialista, Membro do Grupo de Estudos em Atividade Motora Adaptada GEPAMA

Coordenadora da Área de Educação Física da Federação das Entidades Assistenciais de Campinas Fundação Odila e Lafayette Álvaro

\section{Mateus Betanho Campana}

Faculdade de EducaçãoFísica/UNICAMP

Mestrando em Atividade Física, Adaptação e Saúde

Membro do Grupo de Estudos em Atividade Motora Adaptada - GEPAMA

Bolsista- CNPq.

Aprovação no Comitê de Ética - parecer 731/2006 de Dezembro de 2006. 